65.8 per cent.; 47.7 per cent.; 36.1 per cent.; 49.5 per cent. In all but one of these institutions the deaths included all infants under 2 years. As the greatest mortality is under 1 year, the showing would be worse if restricted to this age. The following figures taken from one of these institutions exemplify this point: During 1907, 320 were admitted; of these, 147 died under 1 year and only 18 between 1 and 2 years. During 1908, 417 were admitted, and of these, 113 died under 1 year, and only 12 died between 1 and 2 years.

There is no doubt that the figures cited above give a fair average of the mortality when young infants are collected together in numbers and treated in mass. The high mortality is not so much due to lapses in care or details in management as to the system itself, which fails because it is wrong. As a contributing cause, however, it may be mentioned that rarely, if ever, is sufficient individual care given to infants in institutions. As a result, the condition of those who live is apt to be decidedly below the proper development for the age. It is only fair to say that many babies sent to these asylums are in poor condition from neglect and hardship - sometimes even moribund but still it is equally true that a very much larger proportion could be saved by a different method of handling them.

This consists in placing them in individual homes where the many risks and dangers of the institution will not exist, even though the domicile be crude and rough. The baby will do better here in the long run than in the most immaculate ward, but visiting and watching will be required. The full benefits of boarding out cannot be obtained without proper and continuous oversight, preferably by a trained nurse. This constant supervision is rarely carried out as it should be under the direction of a physician. Thus in one institution, about 700 boarded-out babies are looked after by only four visiting nurses. In this case, it is manifestly impossible to be sure that the babies are receiving the proper medical and hygienic care.

Boarding out must be more systematized and the same detail and routine that are usually employed in institutional work should be applied here. Twelve years ago I instituted a plan of boarding out atrophic or abandoned babies in which the following features were emphasized: (1) Boarding out in a certain district of the country noted for its health ful conditions. (2) Constant attention to diet and hygiene on the part of a physician and nurse who are thoroughly familiar with this class of cases and competent to deal with them. (3) The infants are kept as long as necessary, until feeding is regulated and digestion and assimilation are improved sufficiently to result in an increase in weight. The work is kept up during the whole year and not limited to certain seasons. (4) The training in a given neighborhood of a number of foster mothers, who, by constantly taking these infants into their homes, become fairly expert in handling them under conditions totally unlike those offered by the best institutions and far superior to them. This work has been in successful operation ever since under the control of the Speedwell Society at Morristown, N. J.

This plan can be employed in any place, with such variations as may be required by the special locality. Thus in a large city it may be best to work in distinct districts which can easily be overseen by some one familiar with the neighborhood and its people. This means a more intensive working in many small fields, but all cooperating in a common system. A few small collecting stations that would act as clearing houses can take the place of the large institutions and furnish the doctors and nurses necessary to carrying on the work. A vast field can thus be covered, but the actual work must be done in small units, each represented by a certain district or locality. Relief will thus be instituted along the lines of family life, with individual supervision, instead of the collective life with institutional methods.

The unit of civilization is the family which offers the healthiest physical environment. The most susceptible member of the family to all external conditions is the infant. When transplanted from natural and normal conditions, the little ones quickly droop and suffer most. We must see to it that relief is afforded in the most natural and effective way to these unfortunates who come under our care. For this reason the infant asylum must go. Cottages must take the place of barracks. An increased knowledge of the real needs of infant life will not tolerate the old methods much longer, for a larger and wiser human spirit is at work on these problems, which is not content to put up with evils that can be avoided. If the present workers in this field will not improve their methods, then some future generation with wider vision, truer courage and broader human feeling will accomplish this needed reform.

\section{EXPERIENCES OF THE NEW YORK HEALTH DEPARTMENT IN TYPHOID IMMUNI- ZATION}

L. I. HARRIS, M.D.

Assistant to Director, Bureau of Infectious Diseases

Case Reports by Morris L. Ogan, M.D. Chief, Division of Typhoid Fever NEW YORE

Preventive medicine, despite its brilliant advances in recent days, has met momentary checks, when, as happens from time to time, some apparent instances of its failure to protect from disease have come to light. That element of our lay population which suffers from constitutional timidity and which can be induced only with difficulty, if at all, to adopt the teachings of this newest and most helpful branch of medicine, jumps eagerly at these apparent examples of failure. It makes them serve as justification for relapsing into the helpless and fatalistic attitude toward disease which has come down through the centuries from the dark ages. These particular instances of the so-called miscarriages of prophylactic medicine are trumpeted far and wide and marshaled to serve as evidence against its value, long years after impartial scientists have disproved all criticism and vindicated the signal virtues of this newest department of medical science.

Error and hasty and mistaken conclusions die as hard to-day as ever in the history of scientific progress. In common with every method of treatment that has ever been inaugurated, antityphoid prophylactic treatment has been opposed at each step of its progress by criticism which has sometimes been ignorant, or unscholarly, or malicious, or even all of these. Rus- 
sell has already paid his compliments to these groups. This study of antityphoid immunization is therefore offered to answer unsound criticism as recently expressed in connection with a series of cases that received conspicuous notices in the New York press. Also it aims to give to those general practitioners who have read the necessarily insufficient and confused comments on these cases in the lay press and even in some of the medical journals a knowledge of facts interpreted scientifically, that they may in their praiseworthy office as teachers be reassured and reinforced in their belief in the value of this protective measure, and may impart with renewed enthusiasm and conviction the full measure of their belief to their pupilsthe public.

TIIE EARLY HISTORY OF ANTITYPILOID VACCINATION

Almost simultaneously in 1896, Pfeiffer and Kolle in Germany, and Wright of Netley, England, published the results of experiments in the prevention of typhoid fever by vaccination with Bacillus typhosus killed by heat.

For several years preceding, a group of workers including Dunbar, Fränkel, Simmonds and Stern, had been occupied with the problems of immunology in typhoid fever. Wright made an extensive application of these experiments, vaccinating large groups of men in the British army. His results were not as favorable as had been expected, and the procedure met a setback until Leishman, who had been working with Wright, again essayed the use of this prophylactic measure on a large scale on the men in the Indian service of the British Army. He had concluded that their failure to achieve the success they had anticipated in their first trials was due to a superheated vaccine. This fault was corrected and Leishman's work gave a new impetus to those interested in the subject.

When, subsequently, Wright announced his theory of the negative phase, and the increased risk of infection with typhoid fever, during the period of this phase, progress for a time was again checked. The cumulative, practical experience of Leishman, however, enabled him to refute this theory most emphatically, and to prove conclusively that whatever force it had was more theoretic than real, and "that the most important evidence must come from actual practice, rather than from laboratory experiment." Indeed, Pfeiffer and Friedberger disproved this in laboratory tests on guinea-pigs, as did Leishman in 100 cases at the Baring asylum. Wright himself since 1909 has receded from this position to a very great degree.

In 1911, Major F. F. Russell, than whom there is no more competent authority on this subject in this country, had so far advanced its use among 20,000 men in the United States army that he could assert of those using it that "we knew that the preparation we were using was harmless; we knew too that it caused few severe reactions in healthy persons, and that no vaccination, however severe the immediate reaction may have been, had been followed by permanent injury to the individual; and further that by all laboratory tests the immunity conferred was identical with and equal to that remaining after typhoid fever."* The brilliancy of the results achieved by Major Russell at San Antonio, and subsequently, on an even larger scale in the army, results which now have become almost

* Italics, tire author's. classical, led to the enactment of the compulsory anti typhoid vaccination law by the United States government.

In France, in 19j3, a similar law was passed, making vaccination against typhoid fever compulsory in the French army.

Fully 85 per cent. of the men in the Indian service of the British army have been vaccinated, although no legal compulsion has been employed by the British government.

In the years that have elapsed since 1896, the evidence of the value of this measure is undoubted, and has grown overwhelmingly; so that when the deathrate in the United States was 16.5 per hundred thousand, that in the United States army during 1912 was 0 per hundred thousand. Further examples selected from this accumulated testimony, as they bear on the analysis of our cases, will follow. Well has Russell said, "Our experience since 1909 has left no doubt about the success of antityphoid vaccination in the army and navy. The measure has come to stay."

THE VACCINE-DESCRIPTION AND MODE OF PREPARATION

The prophylactic injections that have been in use have been differently prepared by the various men who have administered them: Leishman in the British army and Russell in America, as well as most other Americans who have employed this procedure, have used a single strain (the Rawlings strain) which is of low virulence. The French prepare a polyvalent vaccine of as many as ten strains, consisting mainly of those found in the neighborhood where an outbreak of typhoid exists. Even in America, some, like Hunt in Troy, $\mathrm{Pa}$., have employed a polyvalent vaccine in which has been included the $B$. paratyphosus $A$, and $B$. paratyphosus B of Gaertner. Needless confusion in the minds of those not familiar with this subject has been caused by the description of these and a variety of other preparations of vaccine.

Metchnikoff and his pupil, Besredka, for instance, have used a vaccine consisting of an emulsion of living typhoid bacilli sensitized by antityphoid serum. Up to 1913 this vaccine, first employed on chimpanzees, was injected into 800 persons in an institution, without any case developing after its use. One soldier in Africa so treated died of typhoid fever, but Metchnikoff explains this as being due to a dose that was entirely too small. Also, forty-eight patients with pronounced typhoid fever were treated with this vaccine. But the consensus of opinion is that aside from the danger of producing carriers, it is far safer to use the sterilized single Rawlings strain of low virulence, which has proved so brilliantly effective in England and the United States.

Chantemesse has produced an antityphoid serum obtained from horses, which has not been approved of by others.

The New York Board of Health vaccine conforms in every particular but one to the army vaccine; the exception being that phenol (carbolic acid) has been substituted for trikresol as a preservative, because of the lessened pain caused by its injection.

The culture used by the Department of Health is prepared at its Research Laboratory and is made as follows:

"A laboratory culture of typhoid bacilli which has lost much of its virulence through long artificial cultivation is used. Large surfaces of agar in Blake bottles are inoculated from fresh agar cultures. After twenty-four hours' growth 
at $37 \mathrm{C}$. the bacteria are washed from the surface of the agar with normal salt solution. The suspension is then standardized by counting the bacilli by the Wright method. This is done by mixing an equal part of blood and the bacterial suspension. Smears are made from this mixture and stained. The number of bacilli and red cells are then counted under the microscope in about twenty-five fields. When the proportion between the two has been determined, the number of red cells per c.c. being known, the number of bacilli per c.c. can then be estimated; this suspension is then heated one hour at $56 \mathrm{C}$., to kill the bacilli. After heating, the sterility of the suspension is tested by inoculating generous amounts into media and incubating these under aerobic and anaerobic conditions. If no growth occurs, 0.25 per cent. of carbolic acid is added to the suspension which is diluted with 0.25 per cent. carbolic acid in normal saline solution so that one c.c. contains the appropriate dose. It is then bottled for distribution."

\section{CASE REPORTS AND COMMENTS}

Whenever we add to the sum of our knowledge and experience, we describe a larger circle to embrace the new as well as the old, and so in this study, even if we contribute little that is essentially new, we believe it well in what follows to include a review of much that has gone before, so that a comprehensive and better correlated picture may the more vividly appear.

In the following are presented the histories of the cases whose atypical features offer several problems of interest, and which have prompted this survey of the work of the New York Department of Health in the field of antityphoid prophylactic treatment.

It will be noted that they fall into four classes:

Class 1. In which death has been attributed to antityphoid inoculation, and was really due to another cause, the prophylactic treatment being a mere coincidence.

Class 2. Severe reactions within a few hours after treatment.

Class 3. In which immunization was partial and insufficient, the patients having been long exposed and already in the stage of incubation when treatment was begun.

Class 4. In which a complete course of prophylactic treatment failed to give immunity, and was followed after a relatively short interval by an attack of the disease.

\section{CLASS 1. COINCIDENCES}

CAss: 1.-C. P., aged, 25, a member of the National Guard, received three injections of antityphoid vaccine in January, 1914. He reacted very moderately to these injections, and for several weeks thereafter continued in good health. Eariy in March, that is, two months after immunization, he became ill and the physician who attended him diagnosed his illness as influenza. Just hefore his death, which occurred March 30 1914, another physician who was called in diagnosed his case as typhoid fever, and so certified in the death certificate he issued.

Acting on the protest of the surgeon of the regiment to which the patient had been attached, the Department of Health refused to accept the death certificate, and a necropsy was therefore ordered.

April 2 the coroner's physician performed the necropsy, and established the absence of typhoid lesions. The hear was found to be the seat of a malignant ulcerative endocarditis. The skin showed petechiae, and septic infarcts were found in various parts of the body, the kidneys particularly.

This case, in which malignant endocarditis ran such a deceptive clinical course that the physicians in attendance were led to believe they were dealing first with influenza and then with typhoid fever is of a type not unfamiliar in clinical medicine and which frequently remains obscure as to diagnosis until it reaches the necropsy table. Such an erroneous charge against typhoid vaccination as this needs no further refutation. The entire lack of logical sequence between vaccination and the ultimate cause of death is clearly evident.

It is reminiscent, however, of the early history of small-pox vaccination, in which, in the case reported by Broeckerhoff, the Jennerian operation was followed by an illness which was held to be small-pox, and because it resulted in death, seemed, until cleared up by necropsy, an aspersion on vaccination, Death in this instance proved to be due to hemorrhage from a typhoid ulcer, and not to the use of vaccine. So once again, it is interesting to note how consistently history repeats itself.

\section{CLASS 2. SEVERE REACTIONS}

Case 2.-A clerk in one of the divisions of the Department of Health was injected one afternoon with antityphoid vaccine, and it was noticed that on withdrawal of the needle a free stream of dark blood followed. This was interpreted to be due to the puncture of a vein. Not more than half an hour later, he was seized with sudden faintness, his skin became pale and clammy, he complained of headache, showed dulness of intellect, and temperature of 103. These symptoms did not last more than a few hours, and the next day he was up and about ready to return to work. The entrance of the vaccine directly into the venous stream was held accountable for the train of symptoms; but no lasting or serious results remained.

Occasionally an urticarial rash on the chest and abdomen, lasting for a week, has been reported, as has also herpes facialis following these injections.

\section{HISTORIES OF CASES 3, 4, 5, 6 AND 7}

Introduction.- $\mathrm{Mr}$. M. arrived at his home in Brooklyn from a business trip through the Southern states, April 23. with a history of illness of ten days' duration. Mr. M.'s physician submitted a specimen of his blood for a Widal test to the New York Department of Health, and on April 25 he was informed that it gave a positive Widal reaction.

At noontime, May 3, in compliance with Mr. M.'s request, the physician administered an immunizing dose of Department of Health vaccine to each of the four presumably well members of the family, consisting of his wife and three children, Ruth, Herbert and Raymond. May 16, Mr. M. died. Mrs. M.'s sister, a Mrs. R., who was present when the prophylactic injections were administered, also received one.

CASE 3.-Mrs. R., the sister-in-law of Mr. M., returned to her home in New York, a distance of at least five miles, and subsequently stated that she had been feeling "very sick" that night. She called no physician to see her. No temperature record or other competent observations were made at the time. She was entirely well in two or three days and able to perform lier regular duties. Her case is mentioned because, in association with the four that follow, it served to inspire the criticism and detraction of a few organs of the lay press and, forsooth, of a medical journal too. In common with Herbert M. and Ruth M., whom Mrs. R. brought to her New York home, five miles distant, directly after they had received prophylactic injections, she made a journey, the length and fatiguing nature of which were contra-indicated after immunizing treatment.

Cases 4, 5, 6 and 7 will be briefly mentioned in this class and again in the next, because they illustrate both the phenomenon of a severe reaction, and the futility of preventive inoculation when administered during the period of incubation.

CASEs 4, 5, 6 AND 7.-These four cases are those of Mrs. $M$. and her three children, two of whom, Ruth, aged $41 / 2$, and Raymond, aged 6 years, made the long trip to New York with their aunt. Within twelve hours after their injection all are reported as having been "very sick," but temperatures were 
not taken, and no more precise and detailed clinical notes are on record as to this particular period than is contained in the foregoing incomplete statement of symptoms. Mrs. M. felt well again within two or three days, and continued nursing her husband. The other children also showed but a temporarily severe reaction, with an intcrval of comparative moderation in symptoms, preceding the conditions to be outlined in that part of the history which is assigned for description in the next class.

In these five cases in which severe reactions were reported to the family physician by the patients themselves, several factors were operative. First, the comparatively long journey to New York made by three of those treated; secondly as will appear from the subsequent history, the $M$. family so treated were not in good health and probably then already in the period of incubation of the disease.

Of this class, characterized by severe reactions that ensue at any time, either immediately after the injection of an immunizing dose of vaccine, or from twelve to forty-eight hours later, much may be said by way of impartial critical comment.

Practically our entire navy has been vaccinated without a single serious result or casualty being noted; and less than 1 per cent. of the men were obliged to rest in bed or be excused from duty as a result of the reaction that followed.

Russell in 128,903 cases observed severe reactions as follows:

After the first dose, 0.3 per cent. in 45,680 .

After the second dose, 0.2 per cent. in 44,321 .

After the third dose, 0.1 per cent. in 38,902 .

Authoritative writers define a severe reaction as one characterized by a temperature of from 103 to 104 , malaise, vomiting, diarrhea and chills.

Spooner, in 400 cases, noted severe reactions in 4 per cent.; moderate reactions, consisting of malaise, headache and a temperature of not above 100.5 in 10 per cent., and very slight or absent reactions in 86 per cent.

Hachtel and Stoner of Baltimore immunized 1,326 persons with an absence of symptoms of distinct reaction in 1,160 , or over 87 per cent.

Malaise appeared in 775 .

Headache appeared in 835.

Muscular pains appeared in 106.

Nausea and vomiting appeared in 60 .

Chills appeared in 57.

Temperature rise appeared in 884.

A study of the temperatures gave the following results:

Temperature normal to 101 in 785 .

Temperature from 101 to 103 in 82 .

Temperature over 103 in 17.

In 1,945 persons on whom they employed a monovalent strain of vaccine, they found that 95.8 per cent. had normal temperature or only a slight rise ; in ninetynine on whom the polyvalent vaccine was used, a normal or slight elevation of temperature was observed in only 80.7 per cent. In other words, severe reactions seem more likely to follow a polyvalent vaccine.

Ravenel of $W$ isconsin vaccinated 1,900 and noted mild reactions in all but two, who after receiving their third injections had drunk heavily.

Weston, in an experience with 898 persons to whom he administered prophylactic treatment, found a severe reaction after the first dose in 883 ; a severe reaction after the second dose in 880 , and a severe reaction after the third dose in 895 .
Of those who received this treatment in the Frencin army, at home and in Africa, severe reactions were noted only in those who were ill or fatigued at the time of the injection, this, despite the fact that a polyvalent vaccine (consisting of ten strains) was employed.

Dr. John W. Brannan, using the New York Department of Health vaccine at Bellevue Hospital on 200 nurses, fifty of the physicians on the house staff, and also on a few male patients in his private practice, reports that of these, only three nurses asked to be relieved of their duties for one day, several interns had a rise of temperature as high as 102 , and one doctor had a temperature of 103 , with headache and abdominal pain after each of two doses.

Rukke of St. Louis, vaccinating 11,007 cases, giving 33,021 injections in all, noted severe reactions in eighty-seven who, in conformity with practice in the United States army, had received simultaneous smallpox vaccinations. Of these eighty-seven, 50 per cent. had vaccinia reactions or reaction from measles or other infectious diseases. Only one case of musculospiral neuritis developed in an officer as the result of trauma to a nerve. He asserts, on the basis of experience in the families of officers living in the camps, that children bear the inoculation well, even at as early an age as 3 years.

Pensuti, using higher doses of vaccine than those generally employed, vaccinated eight children (from 7 to 12 years of age), without subsequent trouble.

All these citations are to show that the occasional occurrence of a severe reaction has been acknowledged and understood by all competent authorities. The severity of these reactions is determined, as already mentioned, by debility from illness (tuberculosis, etc.), marked fatigue, alcoholism or undue exertion in those subjected to prophylactic treatment. In other instances, injection of the vaccine directly into a vein, or its intramuscular administration, has accounted for very marked reactions.

In civil, as distinguished from military communities, whenever a case in which a severe reaction has appeared has been given publicity, it is usually a professional brother who helps spread misinformation with the benerolent suggestion that a contaminated or improperly prepared vaccine has been responsible for such serious result. This charge, if true, is indeed a grave one. Let us examine it: The heating of the suspension of bacilli that is used in preparing the vaccine, at $56 \mathrm{C}$. (132.8 F.) and the subsequent inoculations into mediums incubated under aerobic and anaerobic conditions to test the sterility of the suspension, together with the final addition of 0.25 per cent. phenol, would surely preclude contamination. The only organisms that might then possibly be present as contaminating ones, would be the tetanus bacillus or staphylococcus. The presence of these organisms could surely be recognized - the tetanus organism by the characteristic symptomatology resulting from its action, and the staphylococcus by a localized infection at the site of injection. From the history of the cases thus far given, and as it subsequently appears, it will be clearly apparent that neither of these contaminations of the vaccine were present.

All those who are acquainted with the character and the organization of the Research Laboratory of the New York Department of Health, will feel assured that the vaccine is prepared with every caution and safeguard against contamination. Moreover, Drs. 
Curran, W. S. Hubbard, F. Harnden, C. R. Love and Dudley, of Brooklyn, employed vaccine of the same stock as the foregoing on members of their own families and on private patients without a single severe reaction or untoward result.

CLASS 3. PARTIAL AND INSUFFICIENT IMMUNIZATION

The history of Cases 4, 5, 6 and 7 is resumed under this classification because after the severe reaction caused by the single injection, which each of these patients received, and the short interval of freedom from complaint that ensued, the symptoms of typhoid fever made their appearance in each case.

CASE 5.-Ruth M., aged 4t/2 years, became ill May 7, and was brought back to her home in Brooklyn, from New York, by her aunt. The temperature became high (about 104) and hovered about that point for a number of days, while signs of severe meningeal irritation supervened, that is, marked opisthotonos, stupor, muscular rigidity and loss of sphincter control-justifying the name frequently applied to typhoid, Nervenfieber. Lumbar puncture, performed May 20, proved negative for meningococci, and the spinal fluid was clear and under considerable pressure. A blood count at this time gave the following results: Polymorphonuclears, 41 per cent.; small lymphocytes, 47 per cent.; large -lymphocytes, 9 per cent, mononuclears, 3 per cent.

The Widal test showed agglutination in dilution of $1: 60$ in ten minutes; $1: 120$ in ten minutes; partial agglutination, $1: 500$ in ten minutes; complete in thirty minutes; partial agglutination, $1: 1,000$ in thirty minutes; complete in one hour.

May 22, an examination of the feces showed typhoid bacilli. There was no doubt therefore that this was a case of typhoid fever which became manifest shortly after a single injection of antityphoid vaccine. The patient subsequently made a complete recovery.

CASEs 6 and 7.-The other children, Raymond, aged 6 years, and Herbert, aged 11 years, also developed symptoms of typhoid fever several days after they had recovered from their severe reactions. Raymond's case ran a brief and uneventful course. Herbert had a moderately severe attack of several weeks' duration with a relapse. These two also made complete recoveries.

CASE 4.-Mrs. M., the mother, who had been assiduously nursing her husband until the time of his death, May 16, and then had similarly cared for her children, kept at her work cven wheil initial symptoms of typhoid fever had made their appearance. Like so many ambulatory cases, the date of onset of her illness could not be definitely established. Despite malaise and an increasing feeling of illness she continued her houschold duties and the care of her children until June 13, when her physician reported her ill with typhoid fever. On June 15 she died of intestinal hemorrhage.

Clearly this unfortunate family continuously and intimately in contact with a typhoid fever case for a number of days, before a single vaccine injection was attempted, had incubated the typhoid bacilli too long to make effective the antagonistic and immunizing power of antityphoid vaccination.

A study of the third class of cases assumes special importance, because it raises the question whether it is a safe and proper practice to vaccinate those who are much exposed in an epidemic, or in intimate contact with typhoid fever cases, in the capacity of medical attendant or nurse. Here too, isolated examples are of no value in helping one to arrive at a judgment of the value of prophylactic treatment, and one must perforce utilize all available records from authoritative sources, and from such cumulative evidence derive one's conclusions.

It was in connection with this type of case that Wright offered his theory of the negative phase, and expressed his disapproval of antityphoid inoculations during the period of its presence. His theory and the arguments in its support were based on laboratory experiments, and when they were first promulgated, many excellent clinicians were persuaded by their apparent validity to oppose such prophylactic treatment during epidemics. A characteristic example is McCrae who, in his article on "Enteric Fever" in Osler's "System," said, "It is evident that it would not be wise to vaccinate during an epidemic, as the procedure apparently makes the individual less resistant to infection for a time."

As has already been stated in a previous section, Leishman in a series of practical tests conducted oil 100 individuals exposed at an asylum, made observations that refuted Wright's theoretic views, and in this he was seconded by a goodly number of careful clinicians and laboratory workers. Of the latter, Pfeiffer and Friedbergers' experiments were particularly valuable.

Cullinan of Dublin, a pupil of Wright, inoculated 500 persons at the Richmond asylum during an epidemic of typhoid fever that lasted five months, and only 1.35 per cent. contracted the disease; and those who came down with it were already in the incubation stage. Of the 114 uninoculated nurses, 14.9 per cent. developed typhoid fever.

Spooner inoculated 29 out of 65 persons in a small Vermont town, at a time when 17 had already been brought down by an attack. Of these 29 only one person ( 3.5 per cent.) contracted typhoid fever, whereas of 19 who had refused vaccination, 5 developed the disease-26.3 per cent.

Hunt, who in 1912 took charge of the situation in Troy, Pa., where an epidemic was in progress, and who, judged by his report at the time, is not an enthusiast as to antityphoid prophylaxis, found 1,343 persons who had been definitely exposed. Of 761 who accepted vaccination and were well at the time of the first injection, a total of 37 (4.86 per cent.) developed typhoid fever. Of these 37 cases, 28 occurred after the first injection, 7 after the second and 2 after the third injection. Contrast with this the 582 who were not vaccinated and among whom 65 (14.28 per cent.) came down with enteric fever. Whatever his deductions from the facts observed may have been, his testimony, taken together with that of others, seems strongly to argue for a decided protective action of the vaccine even in the presence of an epidemic. Even his mortality statistics would tend to confirm one's faith in the value of protective inoculations, for, of the 37 cases that appeared among the vaccinated, there was but 1 death ( 0.27 per cent.) as opposed to 17 (8.85 per cent.) among the 65 unvaccinated. Hunt takes issue with Spooner, who believed that there was an acceleration of the onset in the infected vaccinated cases. The former contends that the incubation period was prolonged, and the onset consequently delayed until many days after the second inoculation in four cases, and until seven days after inoculation in another case. The evidence which these particular cases furnish strongly offsets, in Hunt's opinion, the prompt appearance of typhoid fever in 11 cases after the first inoculation.

Nor are these by any means isolated instances in which antityphoid vaccination during epidemics gave an excellent account of itself. It has been recognized that the exposure of hospital attendants, nurses, etc.particularly nurses who do night duty-predisposes them to typhoid fever, in many cases, quite as much 
as would an epidemic. Observations made on this group, therefore, are of telling value. Also it should be borne in mind in this connection that nurses are known to be many times more liable to contract the disease than are the others of a city's population; Joslin and Overlander of Boston have found that the incidence of this disease is from eight to twelve times greater among them; while Hachtel and Stoner found the rate among this group of workers for five years in Baltimore, 500 per ten thousand nurses and hospital attendarits, or from twelve to twenty times greater than in the city generally. Yet at the Massachusetts General Hospital, where immunization was carried out for three years, the morbidity was only 1.4 per cent., or 19 cases in 1,361, even including nurses on night duty. In Baltimore, likewise, the institution of vaccination abolished the disease among the nurses (among 82 nurses during five years who had not been vaccinated, 7 or 8.5 per cent., contracted typhoid fever).

Ravenel, in a small town in which an epidemic was at its height, vaccinated 116 persons, and not one new case appeared thereafter.

Wright and Leishman vaccinated at Maidstone, when an epidemic had attained its maximum intensity, and with success. At Torrington, Conn., of 80 nurses who were employed, 45 accepted vaccination and remained free from the disease, while of 35 who refused the treatment, 3 cases subsequently developed.

Peterson reports that in one house in which the sanitary conditions were of the worst, there were 14 occupants of whom 7 were down with typhoid fever. The remaining 7 were immunized and no further cases developed.

From this summary of statistical data, which could be greatly enlarged on, it must appear that the fear of a negative phase, and the withholding of immuniza. tion from those closely in contact with typhoid fever cases, is not grounded on sound and logical reasoning. The experiences of scientific and conservative observers combine to prove the merit of this prophylactic procedure, and should commend it as a duty to those charged with the care of the community's health, wherever and whenever typhoid fever appears in its midst.

Moreover, the freedom with which conservative clinicians in constantly increasing numbers employ the vaccine as a therapeutic measure, to moderate the severity of this disease when already established, and to diminish its mortality rate, should serve to dispose of any distrust of its effects, which still lingers in some quarters. Wright believes that through its use, the incidence of typhoid fever is diminished about onehalf, and the mortality rate still more. These statements are not to be construed as a support of those who believe in the therapeutic efficacy of the vaccine, for that is still sub judice.

Hornor's studies in the therapeutic employment of the vaccine in 40 of 135 cases, while they show no decided advantage from its use as a curative agent, exhibit, however, no evidence that could in any manner be construed as an argument against the safety of its administration. In addition, the restricted scale on which he has employed it cannot make his experience serve as more than a preliminary experimental note. Compared with Wright's extensive experiences, the limitations of Hornor's report can be better understood.

Russell further reports that in the navy, one mild case followed the second injection. Another man at
Guantanamo, Cuba, received one dose, and two days later complained of headache, had a temperature of 104.6 and manifested dulness of intellect. In twentyfour hours he became rapidly worse. He died seventysix hours after admission to hospital, and seven days after vaccination. The necropsy showed that he was at the beginning of the third week of the disease. The infection was one week old when the single dose of vaccine had been administered. Ambulatory cases are notorious for developing severe symptoms late in the course of the disease, and for their high mortality rate.

The case exemplifies strikingly the manifest difficulty of determining in these exceptional instances whether typhoid infection has already gained a foothold, and is like the cases of Mrs. M. and her daughter Ruth, as recorded in the preceding case reports.

In the Lancet, W. Broughton-Alcock reports the use of living sensitized typhoid bacilli (in contradistinction to the killed bacilli employed by Wright and Russell) in 750 persons, in whom he found it innocuous.

Major Russell in a report in May, 1914, says, "The conclusion is inevitable that the prophylactic vaccine as used in the army has given almost absolute protection against typhoid fever, without producing untoward effects of any character, showing definitely that the vaccination is both efficient and harmless." Those who make the point of their criticism the failure of vaccine to confer absolute immunity, despite the exposure of some patients to exceptionailly large doses of typhoid bacilli, or debilitation which has made the body's resistance to the organism ineffective, are unrelenting and unreasonable judges who will be satisfied with nothing less than perfection. Aside from personal idiosyncrasies which may determine failure to produce antibodies, it must be conceded that exhaustion, or the implantation and growth of a virulent strain of typhoid bacilli may have gained too great a headway to be offset by one or more immunizing doses of vaccine. Even small-pox vaccination, the brilliant achievements of which are accepted by scientists, occasionally fails to confer immunity. Likewise, the administration of diphtheria antitoxin may be deferred so long that the body of the patient is overwhelmed by toxins, and no amount of this effective agent can neutralize the effects of the poison; or, if an antecedent exhausting illness makes the body a ready prey to the virulent diphtheria toxins, it outstrips the neutralizing antitoxin, and the patient succumbs. And yet, though not infallible, diphtheria antitoxin, and possibly, too, the prophylactic use of tetanus antitoxin and antirabic treatment, will continue as our most potent weapons in those diseases. There is scarcely a potent therapeutic measure that has not its limitations or contra-indications.

\section{CLASS 4. FAILURE OF IMMUNITY}

This class embraces the cases in which a course of immunizing treatment has been completed, and in which the patients are, nevertheless, after a relatively short interval brought down with an attack of typhoid fever. Here opponents of this measure have found a fertile field for criticism. And it would seem to those not familiar with the literature of the subject that in this particular a significant and incontrovertible objection to immunization has been advanced.

CASE 8.-Among a group of cases that developed in October, 1913, in a neighborhood on the lower east side, where 
infection had been spread through the milk supply, there were four cases in an Italian family, affecting the mother, a Mrs. C., and three of her children. Through the visits of two married daughters and their children, three additional cases developed in their respective families. A third married daughter, a Mrs. L., whose history illustrates the theme under consideration, also visited quite frequently but was prevailed on to accept prophylactic treatment. Accordingly, two doses of 500 million (killed typhoid) bacilli each, were administered, on October 15 and 25 respectively. November 6,1913 , a final dose of one billion bacilli was injected. These were administered by the Department of Health representatives. All went well with Mrs. L. and her family until March, 1914, when her four children, each in turn came down with typhoid fever, in the following order: Rocco, aged 10, March 10; Theresa, aged 4, March 19; Louis, aged 8, March 19, and Daniel, aged 12, March 29. Subsequent examination of the stools of the grandmother, Mrs. C., at the Research Laboratory, showed typhoid bacilli. She had been the carrier to whom the infection of these cases was traced.

Mrs. L., aged 30, who was about three and a half months pregnant at this time, nevertheless devoted herself assiduously for a number of days to nursing her children and attending to household duties at the same time, until the children were sent to Bellevue Hospital. Despite the fatigue and weakness induced by her overexertions, she continued daily to visit the children at the hospital. (The children ultimately recovered).

April 1, 1914, subjective symptoms of marked lassitude and headache appeared. These Mrs. L. attributed to her pregnancy and gave them no further heed, although they steadily became worse during the second week. At this time sharp cramps developed in the right lower quadrant of the abdomen and persisted despite active catharsis. For four days prior to April 21, her headache, fever, abdominal pain and vomiting grew constantly worse. April 21 she was admitted to the service of Dr. W. Gilman Thompson of the Second Medical Division of Bellevue Hospital. The Widal was positive and a blood count showed 5,000 leukocytes (polymorphonuclears 78 per cent.). The spleen was enlarged and an atypical roseolar eruption was present. April 24, at about the fifth month of gestation, she miscarried, as pregnant typhoid fever patients so often do, but this had no influence on the temperature or the course of the disease. Dr. Thompson from his clinical study of the case diagnosed it as one of typhoid fever. April 28 she died. It is to be regretted that no blood cultures during life, or from the viscera after death, had been made. At necropsy, characteristic typhoid lesions were found in the bowels. Peyer's patches in the ileum were greatly swollen, indurated, red and showed ulceration; the spleen was considerably enlarged.

The case of Mrs. L. is then one of a series in which a debilitated condition (in this case from pregnancy and the worry and fatigue incident to nursing her three children affected with typhoid) together with a prolonged exposure to exceptionally large doses of typhoid bacilli overwhelmed the defensive powers of the individual and allowed the infective agent to become dominant.

Case 9.-Miss E. G., aged 29, a nurse doing settlement work on the lower east side of the city, was immunized in June, 1913, and though frail of body worked very hard, nursing. among many others, several typhoid fever patients. In April, 1914, she again nursed several patients of typhoid - fever, devoting herself with great energy to their care. April 30, 1914-nearly one year after immunization-she noted definitely the onset of malaise, headache and general pains. May 5 she entered the Post-Graduate Hospital with marked febrile symptoms. May 6 a Widal was negative, but a blood culture showed typhoid bacilli. May 11 the Widal was found positive in a dilution of 1:80 (no higher dilutions were attempted). In the meanwhile her temperature at the beginning of the second week showed the typical continuous elevation, varying only between 104 and $105 \mathrm{~F}$. May 20 she died from intestinal hemorrhage.
CASE 10.-Miss D., aged 23, a nurse at the Presbyterian Hospital, received three successive doses of antityphoid vaccine prepared at the hospital, July 1,11 and 22,1913 , respectively. After nursing a particularly severe case she became ill April 27, 1914, with fever, headache, pains in the back and limbs, and sore throat-that is, nine months after immunization. She continued at her work for several days, despite the indisposition caused by the initial symptoms. On May 2 the Widal test was found suggestive in dilution of $1: 20$, and negative in dilution of $1: 100$. Again, May 7, the Widal was positive in a dilution of $1: 20$, and suggestive in $1: 100$. May 13, typhoid bacilli were found in the blood. Her temperature curve was an atypical one showing fluctuation in the second week between 98.8 and 103.5 , reaching 105 at the end of the fourth week, and then continuing its large excursions. After a stormy period of eight weeks she recovered.

Again one turns to the record of Russell's experiences, and there one finds a telling and satisfying answer in quotations from his writings already given, where he says that "by all possible laboratory tests, the immunity conferred was identical with and equal in that remaining after typhoid fever."

To place the problem here presented in different words: If, notwithstanding an attack of typhoid fever, thoroughly authenticated by laboratory and clinical diagnostic tests, there still may follow a second attack of the disease at some later period, even though it is commonly taught and accepted that one attack confers immunity for life, then why should immunity obtained by vaccination be deprecated and tabooed if a like exceptional recurrence follow its practice? That vaccination gives immunity to great masses of those exposed, is most eloquently attested to by the vigorous and severe test it has received in our army and navy among more than 200,000 men. And this should outweigh overwhelmingly the criticism that an occasional exception has evoked. From the literature we shall first cite the reports of recurrences of typhoid fever, and then compare them with the reports of typhoid fever cases subsequent to vaccination, remembering that it is currently held that the latter confers immunity lasting from two to two and one-half years, according to Firth of England. Martha Wollstein, it may here be observed, is quoted as believing in revaccination after one year, though this is contrary to Firth's and Russells belief in a two or two and onehalf years' immunity as noted in their extensive experiences in India and the United States, respectively.

1. Some reports of recurrences are as follows:

Dreschfield in over 2,000 cases at the Hamburg General Hospital reports that fourteen were affected twice, and one three times.

In 500 of Osler's cases in which special inquiry was made as to the history of a previous attack, eleven, or 2.2 per cent., were found in which it had occurred.

W. T. Cummins and P. K. Brown found that of 149 of their cases nine had relapses, one of them occurring four weeks after the patient left the hospital; one other patient developed a second attack after six years.

As to relapses, Strïmpell reports that they occurred in 9 per cent. of all cases of typhoid fever at Leipsic; Murchison, 3 per cent.; Griesniger, 6 per cent., and Shattuck as high as 16 per cent. McCrae in 28,057 collected cases found 2,493 ( 8.8 per cent.); while in 172 of his own cases relapses occurred in 11.4 per cent. Why these relapses should occur is a question at once difficult and not in place here to consider, except to indicate that Durham's explanation of the evolution and unexpected dominance of a heretofore 
subordinate and quiescent strain or family of Bacillus typhosus is quoted with approval by McCrae.

2. Directing our attention to the occurrence of typhoid after a complete course of immunization, we find the following instances recorded in the literature:

Russell reported twenty-seven cases of typhoid occurring among the men of our army and navy, with one death, the result of intestinal hemorrhage. Of these twenty-seven cases of infection in vaccinated persons, four cases developed in less than one month after vaccination; eight cases developed within three months after vaccination; six cases developed within six months, and over three months after vaccination; six cases developed within twelve months, and over six months after vaccination; two cases developed within eighteen months, and over twelve months after vaccination; two cases developed within twenty-four months, and over eighteen months after vaccination; in three cases the interval was unknown.

Here again, the relatively long period elapsing between the time of vaccination and the appearance of infection would argue powerfully against the theory of a negative phase, if further argument were needed.

Hunt, as already mentioned in a different connection, among 761 who were vaccinated, found two in whom typhoid fever followed the third injection.

In the Massachusetts General Hospital, 1,361 were vaccinated, and but two cases of enteric fever developed (a morbidity of 0.15 per cent.) against eight cases in 674 non-immunized persons (a morbidity of 1.19 per cent.). At this institution, of the two cases that developed, both were in nurses, one of whom a year after inoculation cared for two patients outside the hospital, and developed a severe attack, but recovered. The other nurse was free two years after vaccination, but then became infected from typhoid material that accidentally entered her mouth.

Here, then, to summarize the results of this review of the literature, are facts obtained from two different angles, which, brought to a focus, show distinctly that whether it is by an attack of typhoid fever, or by the prophylactic injection of killed typhoid organisms, the immunity acquired by great masses of individuals can be safely depended on, in the one case for a protracted period or even a lifetime, in the other case for a period of from two to two and one-half years or even longer.

When it is seen that in those inoculated, a great decrease in morbidity and mortality as contrasted with uninoculated groups of a population restilts, then indeed is enthusiastic belief in its value justified. Experiences many times multiplied, like those of Wright at Ladysmith, confirm one's faith in its protective power. Of 1,705 persons whon he inoculated, only 2 per cent. contracted typhoid as against 14 per cent. among 10,529 uninoculated persons; and again elsewhere, where of 19,069 inoculated, 266 cases of typhoid ( 1 in 84.4 persons) developed with a mortality of 17 per cent., while among $150,231,3,739$ cases ( 1 in 40 . persons) developed with a mortality of 25 per cent.

No better statement of the present day attitude on the subject of the possibility of obtaining absolute immunity through antityphoid prophylactic treatment could be given than is contained in a letter of Major Russell written from Vera Cruz to the Department of Health, Aug. 10, 1914, and received after this article had been written. We here take the liberty of inserting quotations from it:
As you will note (in the article written in June, 1912, in the American Journal of the Medical Sciences), there was one fatality among the 27 cases, and that from hemorrhage. Twenty of the 27 cases occurred within one year of vaccination, probably because of the greater exposure of recruits to all kinds of infection than occurs among. older soldiers. It is evident that the vaccine cannot be cxpected to give absolute protection, even in the period immediately following it. To be sure, it does protect in almost every instance, but the exceptions are frequent enough to prevent our claiming absolute protection. There is no perfectly satisfactory explanation of the occasional failure to protect. We know, of course, that clinical typhoid is not infrequently followed by relapse, and that the disease may occur twice in the same person. It may be due to an excessive dose of infectious material, as in milk epidemics, or among nurses, or to the apparent impossibility of obtaining a high degree of immunity in certain persons.

I do not believe we are justified in promising absolute immunity even for a short period; we can state, however, that the chances of infection among vaccinated persons is extremely small and that when infection does occur, the prognosis is decidedly better than among the unvaccinated ( 1 death in 27 cases).

We feel very confident that the vaccine protects against the average dose of infectious material, but we have always doubted its power to protect against excessive amounts. While it is true that the protection conferred is not absolute, that is no reason for not using it in dispensaries, hospitals and among contacts as much as possible.

Not a single case of typhoid fever has occurred among the troops with whom I am serving, since they were first mobilized in Texas over two years ago; we are not exposed, however, to milk epidemics to any great extent, since the use of canned milk among troops, while in the field, is almost universal.

It is earnestly desired that all experiences of private physicians in this community, along the lines above indicated, be sent to the department that it may cooperate in their investigation and proper classification, with a view to correlating the mass of testimony to serve for future guidance.

The experiences of the New York Department of Health as here offered indicate that in a civic community no conclusive or valuable study, such as of prophylactic antityphoid injections, is possible, unless the Department of Health can serve as a clearing house to which the facts of isolated experiences of individual physicians are sent, and through which they pass as a well-ordered, logically arranged whole.

\section{GENERAL CONCLUSIONS}

1. The accurate observations recorded in hundreds of thousands of cases leave no doubt as to the preventive powers of antityphoid vaccination in all but a relatively insignificant few.

2. In those subsequently affected it strikingly decreases the morbidity and the mortality.

3 . Severe reactions, if one makes observations from extensive studies (the only correct way), are rare.

4. To avoid severe reactions one must observe carefully several precautions, as follows:

(a) Never administer it to any but the healthy.

(b) To permit of slow absorption, avoid puncture of a vein, or intramuscular injection.

(c) Clean syringe and sterilize the area for injection, using tincture of iodin for the latter purpose.

(d) Children especially are to avoid exposure to the sun following treatment.

(e) Avoid administering it during the menses or pregnancy. 
(f) Allow no hard work or indulgence in alcohol after the injection.

(g) Avoid reinjecting in indurated areas.

5. Severe reactions have never left permanent injury.

6. When the incubation period has begun, the time for antityphoid immunization has passed. The treatment is preventive of typhoid fever, and not a typhoid antitoxin.

7. Long exposure to overwhelming doses of typhoid bacilli (in those who are in close contact with cases and especially in epidemics), may nullify the immunizing powers of antityphoid vaccine, and an attack may therefore follow one or more injections.

8. Chronic illness (tuberculosis, etc.), as well as debility from other causes, and fatigue and exhaustion as well, predisposes to severe reactions.

9. Injections after intimate and long exposure hasten the onset.

10. For a period of at least two years, and possibly more, immunization is as effective in protecting from an attack of typhoid fever as is a previous attack of the disease itself.

11. Recurrences may follow after a complete immunizing course of treatment, in exceptional instances in which debility and fatigue exhaust the resistant and defensive powers of the body, and when exposure to massive doses of typhoid bacilli exists.

Thankful acknowledgment is made of suggestions and assistance received from Dr. John S. Billings, director of the Bureat of Infectious. Diseases of the Department of Health.

1855 Seventh Avenue.

\section{THE PATHOLOGY OF THE MISTAKEN DIAGNOSES IN A HOSPITAL FOR ADVANCED TLBERCULOSIS *}

\section{J. EARLE ASH. M.D.}

BOSTO.

"Progressive medicine recognizes that among the most helpful aids to the advancement of the work of our profession are the critical analysis of errors, either in diagnosis or treatment, the frank discussion of mistakes, and the unremitting investigation of the reasons why procedures in current practice fail." The following confession and analysis of errors is made in this spirit, and further to add its iota of emphasis to the importance of the correlation of clinical and pathologic data. An important purpose of the necropsy is surely lost if it is not observed at least indirectly by the clinician that he may learn of his mistakes or strengthen his convictions.

In the 198 necropsies that have been performed at the Boston Consumptives' Hospital since its foundation, twenty-three cases, or 11.5 per cent., have proved to be non-tuberculous, in so far, at least, that there was no active lesion. The report is not to be construed in any sense as a criticism of the clinical staff of the hospital. It is thoroughly appreciated that under the conditions, in a number of the cases, the diagnosis of tuberculosis was the logical one, and further in their defense it might be stated that seven

* From the Pathological Laboratory of the Boston Consumptives Hospital, Mattapan, Mass.

1. Deaths Following Anestlesia, editorial, The Journal A. M. A. Anril 4. 1914. D. 1098 . of the twenty-three cases they recognized ante mortem as being non-tuberculous and five others were under observation only one, two or three days, so that there was no opportunity for close study. They all had been diagnosed tuberculosis, however, by some physician at some time to account for their reference to this institution.

It is not to be stpposed that this percentage of error extends to all the cases that have been treated in the hospital. It would be lowered by those cases of undoubted tuberculosis that have been discharged otherwise than dead and further, greater effort has been made to obtain permission for necropsy in those cases of special interest and obscurity, among which have been the majority of the non-tuberculous. It does mean, however, that a total of 1,365 hospital days have been usurped by these proved non-tuberculous from the needy tuberculous of the long waiting list that is always a feature of hospitals of this nature. This is a point of considerable economic importance, not so much to general hospitals as to the special institutions receiving sums of money for the specific purpose of attempting to care for the large group of tuberculous humanity.

These cases are not presented in great detail because of their number, but just those points from the clinical and pathologic protocols are given that are of correlative interest including the clinical diagnosis, color, sex and age. They are grouped and briefly discussed on the basis of the chief pathologic findings.

Case 1 (No. 1346).-White woman, aged 48. Clinical diagnosis, acute pericarditis; fibroid tuberculosis. In hospital but one day. Cough and expectoration intermittently for twelve years. Fever, palpitation and precordial distress on admission. Dulness over left apex; few fine, moist râles through left lung; sibilant on right. Heart enlarged to left; friction rub at apex; pulsus paradoxus. Sputum negative for tubercle bacilli.

Necropsy.-Acute fibrinous pericarditis (pneumococci and streptococci); late stage of a resolving pneumonia; dense contracted scar at left apex, 3 by $2.5 \mathrm{~cm}$; mucopus in bronchi; syphilis apparent from changes in aorta and liver and the presence of amyloid histologically.

CASE 2 (No. 1862).-White woman aged 53. Clinical diagnosis, carcinoma and gangrene of lungs. Patient was in hospital but two days. Seven months before, a carcinomatous breast was removed. Cough began one week later. She raised large amounts of fetid, blood-streaked sputum that was negative for tubercle bacilli. There were several frank pulmonary hemorrhages. Dulness over upper portions of both lungs, and lower posterior of right. Numerous and various râles throughout both sides. In addition, there were signs of cavity in right axilla.

Necropsy.-Unresolved and organizing pneumonia; gangrene and abscesses of lungs; bronchopneumonia and encysted empyema at right base. No carcinoma or tuberculosis.

Case 3 (No. 1925).-White man, aged 38. Clinical diagnosis, pulmonary tuberculosis, revised to unresolved pneumonia. Sudden onset, two weeks before admission, with chills, cough and cyanosis. Signs of consolidation over greater portion of left lung. Pericardial friction rub. Sputum ncgative for tubercle bacilli.

Necropsy.-Lobar pneumonia ; empyema (left chest) ; purulent pericarditis.

CASE 4 (No. 2617). - White man, aged 22. Clinical diagnosis, pulmonary tuberculosis; empyema; gangrene of lung; lumbar abscess. For three months suffered loss of weight and strength; severe cough and abundant sputum, positive for tubercle bacilli on one examination. An empyema was 